\title{
Hyperon Radiative Decay
}

\section{Citation}

Kaxiras, Efthimios, Ernest J. Moniz, and Madeleine Soyeur. Hyperon Radiative Decay. Physical Review D 32(3): 695-700.

\section{Published Version}

http://dx.doi.org/10.1103/PhysRevD.32.695

\section{Permanent link}

http://nrs.harvard.edu/urn-3:HUL.InstRepos:2886298

\section{Terms of Use}

This article was downloaded from Harvard University's DASH repository, and is made available under the terms and conditions applicable to Other Posted Material, as set forth at http:// nrs.harvard.edu/urn-3:HUL.InstRepos:dash.current.terms-of-use\#LAA

\section{Share Your Story}

The Harvard community has made this article openly available.

Please share how this access benefits you. Submit a story.

Accessibility 


\title{
Hyperon radiative decay
}

\author{
E. Kaxiras and E. J. Moniz \\ Center for Theoretical Physics, Department of Physics and Laboratory for Nuclear Science, \\ Massachusetts Institute of Technology, Cambridge, Massachusetts 02139
}

\author{
M. Soyeur \\ Centre d'Etudes Nucleaires de Saclay, Service de Physique Theorique, F-91191 Gif-sur-Yvette Cedex, France
}

(Received 2 November 1984)

\begin{abstract}
The radiative decay widths of the low-lying strange baryons are calculated both within the relativistic quark bag model and the nonrelativistic potential model. These widths are found to depend sensitively upon the quark-model dynamics through multiplet mixing and $q^{4} \bar{q}$ admixtures. The comparison between our calculated results and the very limited experimental data is discussed.
\end{abstract}

\section{INTRODUCTION}

With the anticipated development of intense low-energy kaon beams, precision experiments in the energy region corresponding to excitation of the low-lying negativeparity strange baryon resonances will be possible. The $\Lambda\left(\frac{3}{2}^{-} ; 1520\right)$, with a full hadronic width of only $16 \mathrm{MeV}$, is the first resonance above $K N$ threshold and dominates the low-energy $K^{-} p$ cross section; the $\Lambda\left(\frac{1}{2}^{-} ; 1405\right)$ is just below threshold and plays an important role in kaonic atoms. Our aim in this paper is to provide a first calculation of the radiative width of these resonances within the framework of the MIT bag model and to explore the sensitivity of the decay rates to quark-model dynamics. ${ }^{1}$ The narrow widths of these resonances makes them good candidates for studying the radiative decays experimentally, both in free space and inside the nucleus, where the strong interactions will substantially affect resonance propagation.

The study of the $S=-1$ odd-parity baryon resonances in the bag model provides an interesting test of the model. Substantial multiplet mixing is expected for these baryons. This mixing is determined by the mass difference between the $s$ and $u d$ quarks which breaks the SU(3) flavor symmetry and by the hyperfine interaction; it also depends sensitively upon projection of translational degrees of freedom. Orbital excitation and $q \bar{q}$ excitation in the $s$ orbit both lead to odd-parity states; the latter may be especially significant for the $\Lambda\left(\frac{1}{2}^{-} ; 1405\right)$, which is very close to the $K N$ threshold. The bag-model spectroscopy for negativeparity states suffers from an inconsistent treatment of the quadratic boundary condition for local pressure balance for the $p \frac{3}{2}$ orbit; the consequences of this shortcoming will be discussed. We shall see below that the electromagnetic decay rates of the negative-parity $\Lambda$ states depend sensitively on their wave functions, so that measurements of these radiative widths would pose a very interesting challenge for more refined models of baryon structure.

We also present, for comparison, the radiative widths calculated in the nonrelativistic quark model of Isgur and Karl. ${ }^{2,3}$ This model, while suffering the theoretical shortcomings of any quark potential model, has proved enormously successful in correlating hadron spectroscopic and decay data. A comparison between the bag- and potential-model results is interesting in that it provides some indication as to which quantities depend primarily upon the symmetries of the baryon wave functions rather than on the model dynamics. Our nonrelativistic-quarkmodel results in the $S U(6)$ basis agree reasonably well with those of Darewych, Horbatsch, and Koniuk; ${ }^{4}$ we also explore the consequence of symmetry breaking in the orbital wave functions due to the larger strange-quark mass.

The baryon wave functions used in our calculations of the radiative widths are summarized in Sec. II. Section III contains the numerical results and a discussion of the sensitivities displayed. Section IV consists of a summary and concluding remarks.

\section{BARYON WAVE FUNCTIONS}

The radiative-decay scheme under consideration is shown in Fig. 1. We start by describing the baryon wave functions and by commenting on features of particular relevance for our calculations.

The bag-model wave functions are given in the fixedradius static-cavity approximation. ${ }^{5,6}$ The even-parity states consist of $u, d$, and $s$ quarks in the $s \frac{1}{2}$ orbit. The $u, d$ quarks are massless, while the $s$ quark is assigned a mass of $279 \mathrm{MeV}$. The $\Lambda\left(\frac{1}{2}^{+}, 1116\right)$ and $\Sigma\left(\frac{1}{2}^{+}, 1192\right)$ belong to the ground-state SU(3)-flavor octet and the $\Sigma\left(\frac{3}{2}^{+}, 1382\right)$ to the ground-state decuplet. Note that in the $\Lambda\left(\frac{1}{2}^{+}\right)$, the light quarks are coupled to total spin and isospin $J=0$ and $T=0$, while in the $\Sigma\left(\frac{1}{2}^{+}\right)$and $\Sigma\left(\frac{3}{2}^{+}\right)$, they are coupled to ${ }^{7} J=1$ and $T=1$.

The $\Lambda\left(\frac{1}{2}^{-} ; 1405\right)$ and $\Lambda\left(\frac{3}{2}^{-} ; 1520\right)$ wave functions are more complicated. The $q^{3}$ model space for these wave functions in the bag model consists of those configurations with two quarks in the $s \frac{1}{2}$ mode and one quark in the $p \frac{1}{2}$ or $p \frac{3}{2}$ mode. As already mentioned, because of the angular dependence of the $p \frac{3}{2}$ orbit, the quadratic boundary condition of local pressure balance is satisifed only on the average for that mode in the spherical-cavity 


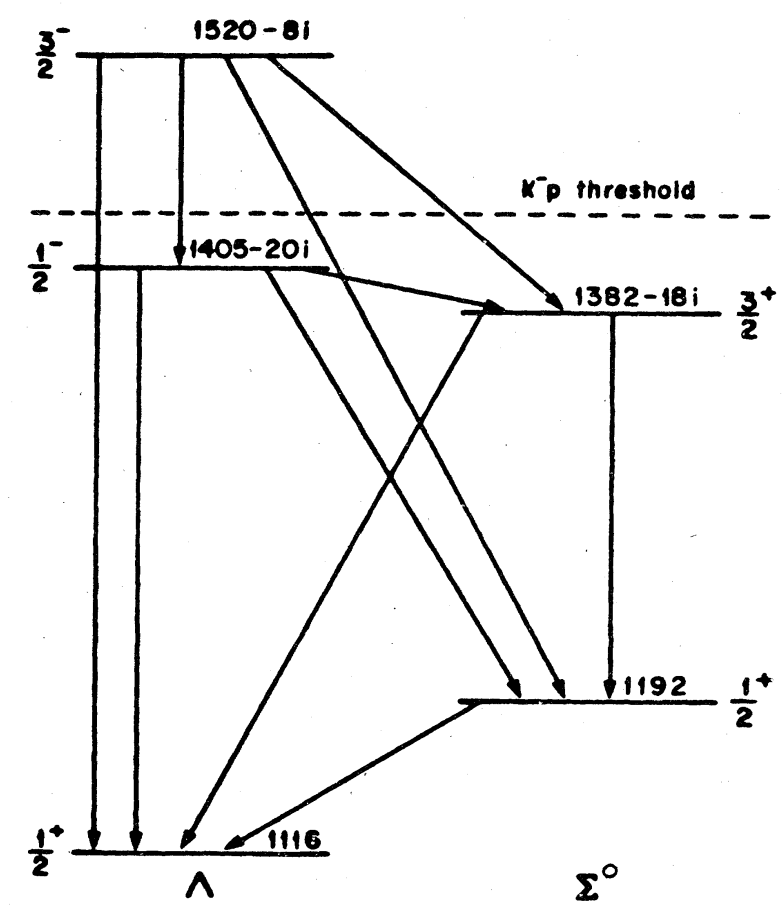

FIG. 1. Radiative-decay scheme for the low-lying neutral hyperons.

approximation. The $p \frac{3}{2}$ energy is then lower than the $p \frac{1}{2}$ energy in apparent conflict with experiment. Another problem arises from the treatment of translational degrees of freedom. In the static-cavity approximation, the bag is not a momentum eigenstate so that the states obtained in this approximation are admixtures of true internal excitations and of translational modes. This problem has been addressed by Rebbi, ${ }^{8}$ who considered small oscillations of the bag about the static boundary. Expressing the $q^{3}$ basis states in $L-S$ coupling as a superposition of $\mathrm{SU}(6) \times \mathrm{O}(3)$ states, he finds that the $L=170$ representation corresponds to the lowest states of bag excitation, while the states in the $L=156$ multiplet correspond either to ground-state translational modes or to true excitations of higher mass. This result has been incorporated in the spectroscopy of the low-lying $p$-wave baryons by projecting the $L=156$ mode out of the $j-j$ coupled basis states. $^{6}$

We consider first the $\Lambda\left(\frac{1}{2}^{-}\right)$wave function. The simplest assumption for the wave function would follow from the observation ${ }^{9}$ that it costs considerably more kinetic energy to promote a light quark to the bag $p \frac{1}{2}$ mode than the strange quark. In this case, the light quarks would be coupled to $S=0, T=0$ and the radiative widths for decay to the $\Sigma\left(\frac{1}{2}^{+}\right), \Sigma\left(\frac{3}{2}^{+}\right)$are zero. This "dynamical selection rule" is broken both by the requirements of translation invariance and by the hyperfine interaction. Using standard MIT bag parameters, ${ }^{5}$ DeGrand ${ }^{6}$ finds the lowest $\Lambda\left(\frac{1}{2}^{-}\right)$ state at $1364 \mathrm{MeV}$ with the wave function (using Lichtenberg phase conventions ${ }^{7}$ )

$$
\begin{aligned}
\Lambda_{1}\left(\frac{1}{2}^{-}\right)= & 0.39 \Lambda\left(1, \frac{1}{2}^{-}\right)+0.42 \Lambda\left(8, \frac{1}{2}^{-}\right)_{A} \\
& +0.46 \Lambda\left(8, \frac{1}{2}^{-}\right)_{B}+0.67 \Lambda\left(8, \frac{3}{2}^{-}\right) .
\end{aligned}
$$

The basis states are labeled by the SU(3) flavor multiplet, by the spin and parity of the excited quark, and, for the octets, by an additional index $A$ or $B$ referring to states totally symmetric or of mixed symmetry, respectively, in pseudospin. ${ }^{9}$ This wave function and those in Eqs. (2) and (4) below are obtained by combining the transformation matrix of Appendix $\mathrm{C}$ and wave functions of Table $I$ in Ref. 6 and expressing the result in the basis of Appendix A in Ref. 9, while accounting for the different phase convention ${ }^{7}$ that we use. It is suprising that this state has a rather small singlet piece. The large $\left(8, \frac{3}{2}^{-}\right)$component presumably reflects the low eigenfrequency of the $p \frac{3}{2}$ mode in the bag. This configuration clearly involves coupling of the $s \frac{1}{2}$ quarks to $S=1$ and thus can couple to the $\Sigma$ states in radiative decay. The second state, predicted at $1446 \mathrm{MeV}$, has the composition

$$
\begin{aligned}
\Lambda_{2}\left(\frac{1}{2}^{-}\right)= & 0.93 \Lambda\left(1, \frac{1}{2}^{-}\right)-0.11 \Lambda\left(8, \frac{1}{2}^{-}\right)_{A} \\
& -0.21 \Lambda\left(8, \frac{1}{2}^{-}\right)_{B}-0.26 \Lambda\left(8, \frac{3}{2}^{-}\right) .
\end{aligned}
$$

This state more closely resembles the $\Lambda\left(\frac{1}{2}^{-}\right)$ground state in the $\mathrm{SU}(3)$ limit suggesting that the first two states are inverted in the bag model. Clearly, a different admixture of flavor multiplets from either of these might emerge from a better treatment. Our approach will be simply to evaluate the radiative decay widths for both $\Lambda_{1}$ and $\Lambda_{2}$ to gain a rough measure of the sensitivity to the $q^{3}$ wave function.

An interesting aspect of the spectroscopy of low-lying odd-parity baryons arises from the possibility that $q^{4} \bar{q}$ configurations are important. Strottman ${ }^{10}$ and Bickerstaff $^{11}$ find that the lighest $q^{4} \bar{q}$ bag state is a $\Lambda\left(^{-}\right)$at about $1400 \mathrm{MeV}$, suggesting that the $\Lambda\left(\frac{1}{2}^{-}, 1405\right) \mathrm{might}$ be a nontrivial superposition of $q^{3}$ and $q^{4} \bar{q}$ configurations. Mulders ${ }^{12}$ investigated this with a $P$-matrix analysis ${ }^{13}$ of the coupled $\pi \Sigma-\bar{K} N$ channels for the SO1 and DO3 waves at energies $\leq 1.75 \mathrm{GeV}$. While the $S$ matrix has two poles in both the $\Lambda\left(\frac{1}{2}^{-}\right)$and $\Lambda\left(\frac{3}{2}^{-}\right)$channels, the $P$ matrix has two $\Lambda\left(\frac{3}{2}^{-}\right)$poles but three $\Lambda\left(\frac{1}{2}^{-}\right)$poles. The two highest $P$-matrix poles seem to form a spin-orbit doublet at 1.71 and $1.74 \mathrm{GeV}$ for the $\Lambda\left(\frac{1}{2}^{-}\right)$and $\Lambda\left(\frac{3}{2}^{-}\right)$ channels, respectively; the spin-orbit splitting of about 30 $\mathrm{MeV}$ is reasonable and the couplings to $\pi \Sigma$ and $\bar{K} N$ channels are roughly the same. ${ }^{12}$ The two remaining $\Lambda\left(\frac{1}{2}^{-}\right)$ poles and the remaining $\Lambda\left(\frac{3}{2}^{-}\right)$pole do not form a spinorbit doublet, leading to the idea that an intruder "primitive," the $q^{4} \bar{q}$ state, has mixed strongly with the spin-orbit partner of the lowest $\Lambda\left(\frac{3}{2}^{-}\right)$state. Using a simple twolevel mixing model, Mulders provides a rough estimate of the relative strength of the two components:

$$
\Lambda\left(\frac{1}{2}^{-}\right) \approx 0.83 \Lambda\left(\frac{1}{2}^{-} ; q^{4} \bar{q}\right) \pm 0.56 \Lambda\left(\frac{1}{2}^{-} ; q^{3}\right) \text {. }
$$

Our estimate of the effect on radiative decay will be based upon a decomposition of the $q^{4} \bar{q}$ component into baryon plus vector-meson components.

The lowest $\Lambda\left(\frac{3}{2}^{-}\right) q^{3}$ bag state ${ }^{6}$ occurs at $1257 \mathrm{MeV}$ 
and has the wave function

$$
\begin{aligned}
\Lambda\left(\frac{3}{2}^{-}\right)= & 0.86 \Lambda\left(1, \frac{3}{2}^{-}\right)+0.35 \Lambda\left(8, \frac{3}{2}^{-}\right)_{S=0} \\
& -0.35 \Lambda\left(8, \frac{3}{2}^{-}\right)_{S=1}-0.14 \Lambda\left(8, \frac{1}{2}^{-}\right) .
\end{aligned}
$$

The index on the $\left(8, \frac{3}{2}^{-}\right)$components indicates the spin of the $\left(s \frac{1}{2}\right)^{2}$ quarks. The low energy of the state is a direct reflection of the low eigenfrequency of the $p \frac{3}{2}$ orbit. The

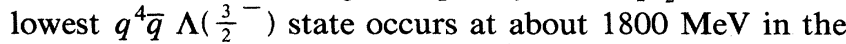
Strottman ${ }^{10}$ and Bickerstaff ${ }^{11}$ calculations, so we assume that the $\Lambda\left(\frac{3}{2}^{-} ; 1520\right)$ is purely $q^{3}$. We note that, in the bag model, the kinetic energy does not favor strange quark excitation to the $p \frac{3}{2}$ orbit. Nevertheless, the $\Lambda\left(\frac{3}{2}^{-}\right) \rightarrow \Sigma\left(\frac{3}{2}^{+}\right)$decay would be zero in the limit of a pure $\Lambda\left(\frac{3}{2}^{-}\right) \mathrm{SU}(3)$ singlet because of the spin structure of the states.

As mentioned above, we shall also present the radiative widths in the nonrelativistic harmonic-oscillator model of Isgur and $\mathrm{Karl}^{2}$ The relevant wave functions are ${ }^{3}$

$$
\begin{aligned}
\Lambda\left(\frac{1}{2}^{+}\right)= & 0.93 \Lambda_{8}{ }^{2} S_{S}-0.30 \Lambda_{8}{ }^{2} S_{S}^{\prime} \\
& -0.20 \Lambda_{8}{ }^{2} S_{M}-0.05 \Lambda_{1}{ }^{2} S_{M} \\
\Sigma\left(\frac{1}{2}^{+}\right)= & 0.97 \Sigma_{8}{ }^{2} S_{S}-0.18 \Sigma_{8}{ }^{2} S_{S}^{\prime} \\
& -0.16 \Sigma_{8}{ }^{2} S_{M}-0.02 \Sigma_{10}{ }^{2} S_{M} \\
\Sigma\left(\frac{3}{2}^{+}\right)= & \Sigma_{10}{ }^{4} S_{S}, \\
\Lambda\left(\frac{1}{2}^{-}\right)= & 0.90 \Lambda_{1}{ }^{2} P_{M}+0.43 \Lambda_{8}{ }^{2} P_{M}-0.06 \Lambda_{8}{ }^{4} P_{M}, \\
\Lambda\left(\frac{3}{2}^{-}\right)= & 0.91 \Lambda_{1}{ }^{2} P_{M}+0.40 \Lambda_{8}{ }^{2} P_{M}+0.01 \Lambda_{8}{ }^{4} P_{M} .
\end{aligned}
$$

The notation $X_{m}{ }^{2 S+1} L_{\sigma}$ corresponds to total spin $S$, orbital angular momentum $L, \mathrm{SU}(3)$-flavor multiplet $m$, and
SU(6)-permutational-symmetry $\sigma$. As discussed by Darewych et al., ${ }^{4}$ a dynamical selection rule similar to that discussed above in the bag model is present in the potential model in the absence of hyperfine splitting; that is, because of the heavier strange quark mass only the strange quark would be orbitally excited in that limit, so that all radiative decay widths for $\Lambda\left(\frac{1}{2}^{-}, \frac{3}{2}^{-}\right) \rightarrow \Sigma\left(\frac{1}{2}^{+}, \frac{3}{2}^{+}\right)$would vanish. The Isgur-Karl wave functions can be expressed in two different bases referred to as SU(6) and uds. ${ }^{3}$ In the latter, the wave function is symmetrized only with respect to exchange of the equal mass quarks. Our results in the SU(6) basis are similar to those of Darewych et $a l ;{ }^{4}$ the differences are due to the fact that in Ref. 4 the ground-state wave functions of $\Lambda\left(\frac{1}{2}^{+}\right)$and $\Sigma\left(\frac{1}{2}^{+}\right)$were approximated by the main component in each case. However, all the SU(6) basis results suffer from an incorrect treatment of the spatial degrees of freedom because the quark masses are taken to be equal. In the $u d s$ basis, we have explicitly taken the strange quark mass into account in determining the hyperon wave functions, specifically, the mass ratio $m_{u, d} / m_{s}=0.6$ leads to a different oscillator frequency for relative motion of the strange quark and $u$-d pair. This consistent treatment of the spatial degrees of freedom will be seen to lead to appreciable changes in the widths for radiative decay of the odd-parity hyperons to the $\Lambda$ ground state.

\section{RADIATIVE DECAY}

With the wave functions described above, the radiative widths are calculated straightforwardly in terms of matrix elements of the electromagnetic current operator. Specifically, the width in the bag model is given by

$$
\begin{aligned}
& \Gamma_{J_{f} J_{i}}=2 k \frac{1}{2 J_{i}+1} \sum_{M_{i} M_{f}} \sum_{\lambda= \pm 1}\left|\left\langle J_{f} M_{f}\left|\int d \mathbf{r} \hat{\boldsymbol{\epsilon}}_{\lambda}^{*}(\mathbf{k}) \cdot \mathbf{j}(\mathbf{r}) e^{-i \mathbf{k} \cdot \mathbf{r}}\right| J_{i} M_{i}\right\rangle\right|^{2}, \\
& \mathbf{j}(\mathbf{r})=e_{f} \psi^{\dagger}(\mathbf{r}) \boldsymbol{\alpha} \psi(\mathbf{r}),
\end{aligned}
$$

where $J_{i} M_{i}, J_{f} M_{f}$ denote the angular momentum and $z$ projection for the inital and final baryons, $\mathbf{k} \equiv k \hat{\mathbf{z}}$ is the photon momentum with $k=\left(m_{i}-m_{f}\right)$ defined by the physical masses, $\hat{\boldsymbol{\epsilon}}_{\lambda}(\mathbf{k})$ is the photon polarization vector, and $e_{f}$ is the flavor-dependent quark charge. The bag radius is taken as $R=1 \mathrm{fm}$. The analogous expression for the nonrelativistic case is

$$
\begin{aligned}
& \Gamma=2 \pi \frac{m_{f}}{m_{i}} \frac{k^{2}}{(2 \pi)^{3}} \frac{4 \pi}{2 J_{i}+1} \sum_{M_{i} \lambda}\left|A_{M_{i} \lambda}\right|^{2}, \\
& H_{\mathrm{int}}=\left[\frac{2 \pi}{k}\right]^{1 / 2}\left[-\frac{e_{j}}{m_{j}} \mathbf{p}_{j} \cdot \hat{\boldsymbol{\epsilon}}_{\lambda}^{*}(\mathbf{k})\right. \\
& \left.+i \frac{e_{j}}{m_{j}} \mathbf{s}_{j} \cdot\left[\mathbf{k} \times \hat{\boldsymbol{\epsilon}}_{\lambda}^{*}(\mathbf{k})\right]\right] e^{-i k \cdot r_{j}},
\end{aligned}
$$

where $e_{j}, m_{j}, \mathbf{p}_{j}$, and $\mathbf{s}_{j}$ are the charge, mass, momentum, and spin of the $j$ th quark and $A$ is the matrix element of $H_{\text {int }}$.

The results, using $q^{3}$ wave functions, are presented in Table I. It is also informative to present the radiative decay widths of the positive parity states as ratios to the $\Sigma^{0}\left(\frac{1}{2}^{+}\right) \rightarrow \Lambda\left(\frac{1}{2}^{+}\right) \gamma$ width. This is shown in Table II. The $\Sigma^{0}\left(\frac{1}{2}^{+}\right) \rightarrow \Lambda\left(\frac{1}{2}^{+}\right) \gamma$ transition is comparatively simple and, as the dominant decay channel for the $\Sigma^{0}\left(\frac{1}{2}^{+}\right)$, has been measured reasonably accurately. The overall scale of the calculated radiative widths is rather sensitive to the parameters chosen, such as the bag radius. With the parameters described above, we obtain widths of $4.6 \mathrm{keV}$ and $8.5 \mathrm{keV}$ in the bag and potential models, respectively, to be compared with the experimental value ${ }^{14}$ of $11.3 \pm 2.6$ $\mathrm{keV}$. Discrepancies of this scale with "standard" parame- 
TABLE I. Radiative decay widths in $\mathrm{keV}$ within each model and experimentally. All calculations use $q^{3}$ wave functions only.

\begin{tabular}{|c|c|c|c|c|c|}
\hline \multirow[b]{2}{*}{ Transition } & \multicolumn{5}{|c|}{ Nonrelativistic model } \\
\hline & \multicolumn{2}{|c|}{ MIT bag model } & SU(6) & $u d s$ & Experiment \\
\hline$\Sigma^{0}\left(\frac{1}{2}^{+}\right) \rightarrow \Lambda\left(\frac{1}{2}^{+}\right) \gamma$ & \multicolumn{2}{|r|}{4.6} & 8.5 & 8.5 & $11.3 \pm 2.6($ Ref. 14$)$ \\
\hline$\Sigma^{0}\left(\frac{3}{2}^{+}\right) \rightarrow \Lambda\left(\frac{1}{2}^{+}\right) \gamma$ & \multicolumn{2}{|r|}{152} & 275 & 273 & $<2100$ (Ref. 16) \\
\hline$\Sigma^{0}\left(\frac{3}{2}^{+}\right) \rightarrow \Sigma^{0}\left(\frac{1}{2}^{+}\right) \gamma$ & \multicolumn{2}{|r|}{15} & 22 & 22 & $<1750$ (Ref. 15) \\
\hline & $\Lambda_{1}\left(\frac{1}{2}^{-}\right)$ & $\Lambda_{2}\left(\frac{1}{2}^{-}\right)$ & & & \\
\hline$\Lambda\left(\frac{1}{2}^{-}\right) \rightarrow \Lambda\left(\frac{1}{2}^{+}\right) \gamma$ & 60 & 17 & 154 & 200 & \\
\hline$\Lambda\left(\frac{1}{2}^{-}\right) \rightarrow \Sigma^{0}\left(\frac{1}{2}^{+}\right) \gamma$ & 18 & 2.7 & 72 & 72 & \\
\hline$\Lambda\left(\frac{1}{2}^{-}\right) \rightarrow \Sigma^{0}\left(\frac{3}{2}^{+}\right) \gamma$ & 1.3 & 0.2 & 0.3 & 0.3 & \\
\hline & $\Lambda_{1}\left(\frac{3}{2}^{-}\right)$ & $\Lambda\left(1, \frac{3}{2}^{-}\right)$ & & & \\
\hline$\Lambda\left(\frac{3}{2}^{-}\right) \rightarrow \Lambda\left(\frac{1}{2}^{+}\right) \gamma$ & 46 & 27 & 98 & 156 & $134 \pm 30$ (Ref. 16) \\
\hline$\Lambda\left(\frac{3}{2}^{-}\right) \rightarrow \Sigma^{0}\left(\frac{1}{2}^{+}\right) \gamma$ & 17 & 102 & 56 & 55 & $<30($ Ref. 17) \\
\hline$\Lambda\left(\frac{3}{2}^{-}\right) \rightarrow \Sigma^{0}\left(\frac{3}{2}^{+}\right) \gamma$ & 3.6 & 0 & 0.03 & 0.07 & \\
\hline$\Lambda\left(\frac{3}{2}^{-}\right) \rightarrow \Lambda\left(\frac{1}{2}^{-}\right) \gamma$ & 0.1 & 0.02 & 0.5 & 0.7 & \\
\hline
\end{tabular}

ters are expected; for example, the radiative widths of the $\Delta^{+}\left(\frac{3}{2}^{+}\right) \rightarrow p \gamma$ transitions are calculated to be $\approx 40 \%$ and $\approx 50 \%$ of the experimental width ${ }^{18}(640 \pm 130 \mathrm{keV})$ in the bag and potential models, ${ }^{19}$ respectively. Given this situation, we feel that the relative magnitudes of Table II are likely to be more meaningful. Decays among the evenparity states are seen from Table II to be practically the same in any of the models, with experiment providing largely useless constraints. Clearly, the wave function in the ground-state octet and decuplet states have the same structure, with differences, such as those in the radial wave functions, affecting mainly the overall scale. A useful check of the quark model would be provided by experimental measurement of the $\Sigma^{0}\left(\frac{3}{2}^{+}\right) \rightarrow \Lambda\left(\frac{1}{2}^{+}\right) \gamma$ width which, like the $\Delta^{+}\left(\frac{3}{2}^{+}\right) \rightarrow p \gamma$ width, is expected to be large.

Octet admixtures play a very important role in the odd-parity transitions. The $\Lambda\left(\frac{3}{2}^{-}\right)$and $\Lambda_{2}\left(\frac{1}{2}^{-}\right)$wave functions are dominated by singlet components. The $\Lambda\left(\frac{3}{2}^{-}\right)$decays given a pure $\Lambda\left(1, \frac{3}{2}^{-}\right)$configuration are given in Table $\mathrm{I}$; the analogous result for the $\Lambda\left(1, \frac{1}{2}^{-}\right) \rightarrow \Lambda\left(\frac{1}{2}^{+}\right) \gamma$ decay in the bag model is $41 \mathrm{keV}$, compared to the $\Lambda_{2}$ results of $17 \mathrm{keV}$. Clearly, the octet effects are very large. These octet admixtures arise from hyperfine interactions, from differences in quark masses and from projection of the translational degree of free-

TABLE II. Radiative decay widths of the positive-parity states; ratios to the $\Sigma^{0}\left(\frac{1}{2}^{+}\right) \rightarrow \Lambda\left(\frac{1}{2}^{+}\right) \gamma$ width.

\begin{tabular}{cccc}
\hline \multicolumn{1}{c}{ Transition } & MIT bag model & SU(6) & $u d s$ \\
\hline$\Sigma^{0}\left(\frac{1}{2}^{+}\right) \rightarrow \Lambda\left(\frac{1}{2}^{+}\right) \gamma$ & 1 & 1 & 1 \\
$\Sigma^{0}\left(\frac{3}{2}^{+}\right) \rightarrow \Lambda\left(\frac{1}{2}^{+}\right) \gamma$ & 33.0 & 32.3 & 32.1 \\
$\Sigma^{0}\left(\frac{3}{2}^{+}\right) \rightarrow \Sigma^{0}\left(\frac{1}{2}^{+}\right) \gamma$ & 3.3 & 2.6 & 2.6 \\
\hline \hline
\end{tabular}

dom. Similar conclusions can be drawn from the nonrelativistic results.

The $\Lambda\left(\frac{3}{2}^{-}\right) \rightarrow \Lambda\left(\frac{1}{2}^{+}\right) \gamma$ radiative width has been measured by two groups ${ }^{16,17}$ with very different results. Mast et $a l .{ }^{16}$ found a branching ratio of $0.86 \pm 0.14 \%$ giving a radiative width of $134 \pm 30 \mathrm{keV}$. This experimental width was obtained with a model-dependent, appreciable $\Sigma^{0} \gamma$ background subtraction assuming that

$$
\Lambda\left(\frac{3}{2}^{-}\right) \rightarrow \Lambda\left(\frac{1}{2}^{+}\right) \gamma / \Lambda\left(\frac{3}{2}^{-}\right) \rightarrow \Sigma^{0}\left(\frac{1}{2}^{+}\right) \gamma \approx 0.4 ;
$$

this ratio is close to that expected for a pure singlet. Both the bag- and the potential-model calculations predict a very different ratio, $\approx 2$ to 3 , because of octet admixtures, and thus cast doubt on the background-subtraction procedure. A recent measurement by Bertini et al. ${ }^{17}$ gives an upper bound for the $\Lambda\left(\frac{3}{2}^{-}\right) \rightarrow \Lambda\left(\frac{1}{2}^{+}\right) \gamma$ transition in disagreement with the earlier measurement. The experimental situation must be clarified before any conclusions can be drawn. Because of the uncertainties in overall scale underlined in our discussion of the positive-parity states, it should be emphasized that numerical agreement between theory and experiment for one transition should not be taken as definitive in favor of a given model. Clearly, the ratio

$$
\Lambda\left(\frac{3}{2}^{-}\right) \rightarrow \Lambda\left(\frac{1}{2}^{+}\right) \gamma / \Lambda\left(\frac{3}{2}^{-}\right) \rightarrow \Sigma^{0}\left(\frac{1}{2}^{+}\right) \gamma
$$

depends very sensitively upon the quark dynamics; an experimental value will be available upon further analysis of the Bertini et al. ${ }^{17}$ data.

The $\Lambda\left(\frac{3}{2}^{-}\right) \rightarrow \Sigma^{0}\left(\frac{3}{2}^{+}\right) \gamma$ decay is very small in the potential model, reflecting the "selection rule" described above. The bag calculation, however, yields a width comparable to the $\Sigma^{0}\left(\frac{1}{2}^{+}\right) \rightarrow \Lambda\left(\frac{1}{2}^{+}\right) \gamma$ width, providing a difficult but interesting experimental test.

As discussed in Sec. II, the $\Lambda\left(\frac{1}{2}^{-}\right)$case is the most complicated. The results for $\Lambda_{1}$, and $\Lambda_{2}$ in the bag model 
and for the potential model are all quite different, although the ratio

$$
\Lambda\left(\frac{1}{2}^{-}\right) \rightarrow \Lambda\left(\frac{1}{2}^{+}\right) \gamma / \Lambda\left(\frac{1}{2}^{-}\right) \rightarrow \Sigma^{0}\left(\frac{1}{2}^{+}\right) \gamma
$$

is similar in all cases. Recall that $\Lambda_{1}$ is suspected to be a poor representation of the $q^{3} \Lambda\left(\frac{1}{2}^{-} ; 1405\right)$ wave function; the factor of $\approx 4$ to 5 suppression with $\Lambda_{2}$ could thus supply insight into the structure of this resonance. This conclusion is somewhat weakened by the complication introduced by $q^{4} \bar{q}$ components [see Eq. (3)]. The contribution of the $q^{4} \bar{q}$ component to radiative decay is evaluated as above except that the current operator, Eq. (11), now annihilates a spin-triplet, color-singlet $q \bar{q}$ pair in the $s \frac{1}{2}$ orbit (this is essentially the vector-dominance model). Bickerstaff $^{11}$ finds very small probabilities, $\approx 0.1 \%$ for dissociation of the $\Lambda\left(\frac{1}{2}^{-} ; 1405\right)$ into $\Lambda\left(\frac{1}{2}^{+}\right) \omega_{0}$ and $\Sigma\left(\frac{1}{2}^{+}\right) \rho$ (as pointed out by Bickerstaff, ${ }^{11}$ Strottman ${ }^{10}$ gets much larger dissociation probabilities because of a phase inconsistency). These small probabilities lead to small widths from the $q^{4} \bar{q} \Lambda\left(\frac{1}{2}^{-}\right)$states; for example, we get $0.8 \mathrm{keV}$ for the $\Lambda\left(\frac{1}{2}^{-}\right) \rightarrow \Sigma\left(\frac{1}{2}^{+}\right) \gamma$ decay. Consequently, the main effect of the $q^{4} \bar{q}$ component is to decrease the $\Lambda\left(\frac{1}{2}^{-}\right)$width by reducing the $q^{3}$ probability. With Mulder's $P$-matrix analysis, ${ }^{12}$ the reduction is about a factor of 3 .

\section{CONCLUDING REMARKS}

We have calculated the radiative widths of the lowlying neutral hyperons. These may be studied experimentally at kaon factories with intense low-energy kaon beams, using the $\Lambda\left(\frac{3}{2}^{-} ; 1520\right)$ as a "doorway." Other potentially interesting radiative transitions between lowmass hyperons are discussed briefly in the Appendix (the charged-hyperon transitions may be studied experimentally using Primakoff excitation with hyperon beams at high-energy accelerators ${ }^{20}$ ). In the limit in which only the strange-quark mass breaks the SU(3) flavor symmetry, a number of selection rules emerge, both in the relativistic bag and nonrelativistic potential models. However, only a trace of these survive the multiplet mixing induced by hyperfine interactions and imposition of translation invariance. Nevertheless, we see that the radiative widths can present an important challenge to our understanding of baryon structure; for example, (i) the $\Lambda\left(\frac{3}{2}^{-}\right)$ $\rightarrow \Lambda\left(\frac{1}{2}^{+}\right) \gamma / \Lambda\left(\frac{3}{2}^{-}\right) \rightarrow \Sigma^{0}\left(\frac{1}{2}^{+}\right) \gamma$ ratio is radically altered by the predicted octet components in the $\Lambda\left(\frac{3}{2}^{-}\right)$and (ii) the $\Lambda\left(\frac{1}{2}^{-}\right)$decay rates in the bag model are strongly suppressed when the dominantly singlet (i.e., $\Lambda_{2}$ ) configuration is used instead of the lowest state (which may be unrealistic because of the low $p \frac{3}{2}$ eigenfrequency). A further large suppression of the $\Lambda\left(\frac{1}{2}^{-}\right)$radiative decays would signal substantial $q^{4} \bar{q}$ components, which in turn would help to clarify the spectroscopic situation. Thus, sensitivity of these decay rates to the quark dynamics is evident, but experimental data for the set of transitions indicated in Fig. 1 are needed to guide theoretical efforts for better understanding the structure of the low-lying oddparity hyperons. Further measurements of these radiative widths inside nuclei may then shed light on the hadronic interactions of these odd-parity hyperons, providing interesting tests of theories of low-energy hadron dynamics.

\section{ACKNOWLEDGMENTS}

One of us (M.S.) thanks Paul Bickerstaff, Tom DeGrand, Robert Lacaze, and Dan Strottman for helpful comments. She also acknowledges the hospitality of the Theoretical Summer Institute of the University of Washington where part of this work was done. This work was supported in part through funds provided by the U.S. Department of Energy under Contract No. DE-AC0276ERO3069.

\section{APPENDIX}

We present here radiative widths for other decupletoctet even-parity transitions and for $\Omega^{-}$decay. We have in mind the possibility that charged hyperon radiative decay may be measured by Primakoff excitation of veryhigh-energy charged hyperons in the Coulomb field of a heavy nucleus. The composition of the $S=-2$ and $S=-3$ hyperons are taken simply as

$$
\begin{aligned}
& \Xi\left(\frac{1}{2}^{+} ; 1325\right)=\Xi_{8}{ }^{2} S_{s}, \\
& \Xi\left(\frac{3}{2}^{+} ; 1530\right)=\Xi_{10}{ }^{4} S_{s}, \\
& \Omega\left(\frac{3}{2}^{+} ; 1675\right)=\Omega_{10}{ }^{4} S_{s}, \\
& \Omega\left(\frac{3}{2}^{-} ; 2020\right)=\Omega_{10}{ }^{2} P_{M}, \\
& \Omega\left(\frac{1}{2}^{-} ; 2020\right)=\Omega_{10}{ }^{2} P_{M} .
\end{aligned}
$$

The potential-model results are given in Table III [the SU(6) and $u d s$ results are almost identical]. We give only the results which are not forbidden by $U$-spin conservation in lowest order [the $\Sigma^{-}\left(\frac{3}{2}^{+}\right) \rightarrow \Sigma^{-}\left(\frac{1}{2}^{+}\right) \gamma$ and $\Xi^{-}\left(\frac{3}{2}^{+}\right) \rightarrow \Xi^{-}\left(\frac{1}{2}^{+}\right) \gamma$ widths are nonzero, $\approx 3 \mathrm{keV}$, due to octet-decuplet mixing]. A systematic study of the radiative widths for the $S=-2$ and $S=-3$ hyperons, together with the $\Lambda, \Sigma$ results, would help to isolate strange quarks mass effects in the baryon structure. We also note that the odd-parity $\Omega^{-}$states have not been identified yet. Given the calculated radiative width, we expect a Primakoff cross section

$$
\begin{aligned}
& \frac{d \sigma}{d \Omega} \approx\left[Z F(q)\left(\frac{E_{f}}{100 \mathrm{GeV}}\right] \frac{2 \delta \theta}{\delta^{2}+\theta^{2}}\right]^{2} \frac{\mathrm{nb}}{\mathrm{sr}}, \\
& \delta=\left(\frac{0.64 \mathrm{GeV}}{E_{f}}\right]^{2},
\end{aligned}
$$

where $Z$ and $F(q)$ are the nuclear charge and form factor, respectively, and $E_{f}$ is the final energy.

TABLE III. Radiative widths for $U$-spin-allowed transitions in the potential model.

\begin{tabular}{lc}
\hline \hline \multicolumn{1}{c}{ Transition } & Width (keV) \\
\hline$\Sigma^{+}\left(\frac{3}{2}^{+} ; 1385\right) \rightarrow \Sigma^{+}\left(\frac{1}{2}^{+} ; 1192\right) \gamma$ & 117 \\
$\Xi^{0}\left(\frac{3}{2}^{+} ; 1385\right) \rightarrow \Xi^{0}\left(\frac{1}{2}^{+} ; 1325\right) \gamma$ & 135 \\
$\Omega^{-}\left(\frac{3}{2}^{-} ; 2020\right) \rightarrow \Omega^{-}\left(\frac{3}{2}^{+} ; 1675\right) \gamma$ & 18 \\
$\Omega^{-}\left(\frac{1}{2}^{-} ; 2020\right) \rightarrow \Omega^{-}\left(\frac{3}{2}^{+} ; 1675\right) \gamma$ & 18 \\
\hline \hline
\end{tabular}


${ }^{1} \mathrm{~A}$ preliminary version of some of these results was presented in E. J. Moniz and M. Soyeur, in Proceedings of the Kaon Factory Workshop, Vancouver, 1979, edited by M. K. Craddock (TRIUMF Report No. TRI-79-1), p. 67.

${ }^{2}$ N. Isgur and G. Karl, Phys. Rev D 18, 4187 (1978); 19, 2653 (1979).

${ }^{3}$ R. Koniuk and N. Isgur, Phys. Rev. D 21, 1868 (1980).

${ }^{4}$ J. W. Darewych, M. Horbatsch, and R. Koniuk, Phys. Rev. D 28, 1125 (1983).

${ }^{5}$ A. Chodos, R. L. Jaffe, K. Johnson, C. B. Thorn, and V. F. Weisskopf, Phys. Rev. D 9, 3471 (1974); A. Chodos, R. L. Jaffe, K. Johnson, and C. B. Thorn, ibid. 10, 2599 (1974); T. DeGrand, R. L. Jaffe, K. Johnson, and J. Kiskis, ibid. 12, 2060 (1975).

${ }^{6}$ T. A. DeGrand, Ann. Phys. (N.Y.) 101, 496 (1976).

${ }^{7}$ D. B. Lichtenberg, Unitary Symmetry and Elementary Particles (Academic, New York, 1978).

${ }^{8}$ C. Rebbi, Phys. Rev. D 12, 2407 (1975); 14, 2362 (1976).
${ }^{9}$ T. A. DeGrand and R. L. Jaffe, Ann. Phys. (N.Y.) 100, 425 (1976).

${ }^{10}$ D. Strottman, Phys. Rev. D 20, 748 (1979).

${ }^{11}$ R. P. Bickerstaff, University of Melbourne Report No. UMP-82/22 (unpublished); Phys. Rev. D 25, 279 (1981).

12P. J. Mulders, contribution to Workshop on Nuclear and Particle Physics at Energies up to $31 \mathrm{GeV}$, Los Alamos, 1981 (unpublished).

${ }^{13}$ R. L. Jaffe and F. E. Low, Phys. Rev. D 19, 2105 (1979).

${ }^{14}$ F. Dydak et al., Nucl. Phys. B118, 1 (1977).

15J. Colas et al., Nucl. Phys. B91, 253 (1975).

${ }^{16}$ T. S. Mast et al., Phys. Rev. Lett. 21, 1715 (1968).

${ }^{17} \mathbf{R}$. Bertini et al., contribution to the International Conference on Particles and Nuclei, Heidelberg, 1984 (unpublished).

${ }^{18}$ F. A. Berends and D. L. Weaver, Nucl. Phys. B30, 575 (1971).

${ }^{19}$ K. Chao, N. Isgur, and G. Karl, Phys. Rev. D 23, 155 (1981).

${ }^{20}$ M. V. Hynes (private communication). 